\title{
Dynamics of Paramagnetic Squares in Uniform Magnetic Fields
}

\author{
Di Du, Peng He, Yongchao Zeng, Sibani Lisa Biswal* \\ Department of Chemical and Biomolecular Engineering \\ Rice University, Houston, TX 77005
}

\author{
*CORRESPONDING AUTHOR: \\ Sibani Lisa Biswal \\ 6100 Main St. MS 362 \\ Houston, TX 77005 \\ 713-348-6055 \\ biswal@ rice.edu
}

\begin{abstract}
The magnetic forces between paramagnetic squares cannot be calculated using a classic dipolar model because the magnetic field distribution is not uniform within square particles. Here, we present the calculation of magnetic forces and torques on paramagnetic squares in a uniform 2-D magnetic field using a Laplace's equation solver. With these calculations, we simulate the variations in equilibrium configurations as a function of number of interacting squares. For example, a single square orients with its diagonal directed to the external field while a system of multiple squares will assemble into chain-like structures with their edges directed to the external field. Unlike chains of spherical magnetic particles, that easily stagger themselves to aggregate, chains consisting of magnetic squares are unable to aggregate due to interchain repulsion.
\end{abstract}




\section{Introduction}

The manipulation of superparamagnetic colloidal particles using magnetic field has been employed in a variety of applications, such as altering fluid rheology [1, 2], directed motion [3, 4], force probing [5, 6] as well as model systems for investigating physical phenomena like phase behavior $[7,8]$. In these applications, the colloidal particles are typically spherical in shape and their mutual interaction forces are classically calculated by dipole-based models [9]. The basis of dipole-based models is that in the presence of a uniform magnetic field, a spherical paramagnetic particle acquires uniform magnetization and thus generates a dipolar magnetic field around the particle [10]. Predictably, dipole-based models cannot be used to calculate the magnetic forces between non-spherical magnetized bodies. Alternatively, these forces can be calculated more accurately using a Maxwell stress tensor combined with solving Laplace's equation for magnetostatics [11], which is referred to as the Laplace's equation solver (LES) method. This method is inherently capable of calculating the magnetic force on any enclosed magnetized body, thus it is not restricted by shape.

With new interesting synthesis methods, colloidal squares are easily realized. Square particles show interesting packing behavior and have been reported as a model system for molecular liquid crystals [12-14]. Most recently, an analytical study on ferromagnetic squares situated periodically on a cubic lattice site has been reported [15]. However, there are few reports that describe the ability to tune the interaction potential between square colloids or describe their assembly dynamics. Here, we perform calculations of the magnetic force and torque on arbitrarily placed paramagnetic squares in a uniform magnetic field using the LES method. The squares or rectangles used in this work have a short edge length $D=2 \mu \mathrm{m}$, a volumetric magnetic susceptibility $\chi=1$, and an external magnetic field strength with a magnitude of 
$B_{0}=10$ Gauss in the positive $\mathrm{x}$-axis direction. These calculations reveal how particles with four-fold symmetry aggregate in a uniform magnetic field.

\section{Numerical Methods}

In the perspective of scalar magnetic potential, the Maxwell equations for magnetostatics can be simplified to Laplace's equations for different media [11]:

$$
\begin{aligned}
& \nabla^{2} \phi_{i}=0 \\
& \nabla^{2} \phi_{D M}=0
\end{aligned}
$$

where $\phi_{i}$ is the scalar magnetic potential for particle $n$, and $\phi_{D M}$ is the magnetic potential for the dispersion media. The boundary conditions at the surfaces of the squares are

$$
\begin{aligned}
& \phi_{i}=\phi_{D M} \\
& \mu_{p} \frac{\partial \phi_{i}}{\partial r_{i}}=\mu_{0} \frac{\partial \phi_{D M}}{\partial r_{i}}
\end{aligned}
$$

where $\mu_{p}=\mu_{0}(1+\chi)$ is the particle permeability. The boundary condition at $r_{i} \rightarrow \infty$ is

$$
\phi_{D M}=-\mathbf{H}_{0} \cdot \mathbf{r}_{i}
$$

Due to the complex boundary conditions, the profile of permeability is smoothed using indicator functions [11]. The indicator function $\lambda_{i}(\mathbf{r})$ for the $i$ th particle is

$$
\lambda_{i}(\mathbf{r})=\frac{1}{2}\left[\min \left(\tanh \left(\frac{1-\left|r_{i x}^{\prime}\right|}{\xi}\right), \tanh \left(\frac{1-\left|r_{i y}^{\prime}\right|}{\xi}\right)\right)+1\right]
$$


Here $\xi$ is the thickness of the interface between the particle and the surrounding medium, $r_{i x}^{\prime}$ and $r_{i y}^{\prime}$ are the $x$ and $y$ components of $r_{i}^{\prime}=M \cdot\left(r-r_{c, i}\right)$ where $r_{c, i}$ is the central position of the $i$ th particle, and $M$ is the orientation matrix defined as $M=\left(\begin{array}{ll}\cos \theta & \sin \theta \\ -\sin \theta & \cos \theta\end{array}\right)$ where $\theta$ is the orientation angle between the edge of the square and the external magnetic field. An overall indicator function for the entire system is given by summing up the indicator functions over all particles.

$$
\lambda(\mathbf{r})=\sum_{i=1}^{n} \lambda_{i}(\mathbf{r})
$$

The discontinuous physical property in the equation is in the permeability $\mu$, therefore the overall indicator function is used to smooth the profile of it.

$$
\frac{1}{\mu(\mathbf{r})}=\frac{1}{\mu_{p}(\mathbf{r})} \lambda(\mathbf{r})+\frac{1}{\mu_{0}(\mathbf{r})}(1-\lambda(\mathbf{r}))
$$

By using the smoothed profile, the boundary conditions at the interfaces will be automatically satisfied. A two-grid method is used to numerically solve smoothed Eqn. (1) [11].

\section{Results And Discussions}

\subsection{A single square}

In two-dimensions (2-D), paramagnetic spheres can be considered as disks, which also acquire uniform magnetization when placed in a uniform magnetic field. However, paramagnetic squares do not have a uniform magnetic field distribution within the particle (Figs. 1(a) and 1(b)) when 
placed in a uniform magnetic field. Additionally the magnetic field distribution outside the square is not axisymmetric since the symmetry axis of the square does not coincide with the orientation of the external magnetic field. The highest magnetic field intensity is distributed in the vicinity of the far-left and the far-right vertices of the square, leading to a non-zero torque rotating the square towards an orientation where its long axis coincides with the direction of the external field. Figure 1(c) shows the torque $\tau$ exerted on the square for different edge-to-field angles, $\theta$. We set the initial angle $\theta=0^{\circ}$ for our calculations. When $-45^{\circ}<\theta<0^{\circ}$, the torque is counterclockwise (Fig 1(b)), changing $\theta$ towards $-45^{\circ}$, to the orientation where $\tau=0$. When $0^{\circ}<\theta<45^{\circ}$, the torque is clockwise (Fig 1(a)), changing $\theta$ towards $45^{\circ}$ where $\tau=0$. This indicates that at $\theta=0^{\circ}$ the configuration of the square is metastable. Though $\tau=0$ at $\theta=0^{\circ}, \lim _{\theta \rightarrow 0^{+}} \tau=2.6 \cdot 10^{-20} \mathrm{~N} \cdot \mathrm{m}$ and $\lim _{\theta \rightarrow 0^{-}} \tau=-2.6 \cdot 10^{-20} \mathrm{~N} \cdot$ $\mathrm{m}$, leading to a singularity at $\theta=0^{\circ}$. Therefore a paramagnetic square always orients along its diagonal in the direction of the external field.

\subsection{Two squares}

Considering the findings for a single square, when another square is added into the system, each square tries individually orient its diagonal into the direction of the external field. However, as they approach each other in close contact, their configuration is influenced by the high magnetic field distribution near each other vertices. In order to predict the final stable configuration, the forces and torques on the two squares must be analyzed (Fig. 2). For practical realization, most colloids typically have an electrostatic repulsive interaction potential. We consider $\mathbf{F}_{\text {mag }}$, the magnetic attractive force and $\mathbf{F}_{\text {ele }}$ for the electrostatic repulsive force. Here we assume the 
squares are physically separated by a finite quantity by surface charge repulsion and thus the Maxwell stress tensor can be applied to calculate the force without considering interfacial contact between the squares [11]. We further assume that the distance between the facing edges is fixed to $0.1 D$, which is valid as the particle distance does not significantly change when balancing the magnetic and electrostatic forces [8]. The alignment length of the edges is defined as $\varepsilon \lambda D$, where $\lambda$ is the alignment ratio and $\varepsilon$ is the aspect ratio (for squares $\varepsilon=1$ ).

The motion of the two squares can be simulated using the forces calculated using the LES method. Here the two squares are initiated with an orientation angle $\theta=45^{\circ}$ and $\lambda=0.1$. For simplicity, we assume Newtonian dynamics without considering the interaction between squares and the surrounding media. The inclusion of hydrodynamic interactions will certainly alter the dynamics of the system but the thermodynamics will dictate the final configuration. Figure 3(a) shows that the stable configuration for two squares is when they align with their edges, which differs completely from that of a single square. The dynamics show that the orientation of the connector vector, defined as the line connecting the centers of the squares, prefers to align the squares to be $\theta=0^{\circ}$. Changes in the orientation and alignment ratio of the two squares from an unfavorable initial position to a stable one are shown in Figs. 3(b) and (c). The elapsed time can be made dimensionless by scaling it with magnetic field strength $B_{0}$, volumetric susceptibility of the material $\chi$, and edge length $D$ using the following equation:

$$
t^{\prime}=\frac{\chi^{2} B_{0}^{2}}{\varepsilon c \rho \mu_{0} D^{2}} t
$$

where $\rho$ is the density of the square, $c$ the speed of light and $\mu_{0}$ the vacuum permeability. The scalability of the elapsed time in this approach is confirmed by the four sets of data points in these two figures, which correspond to the cases of doubling each scaling parameter and regular 
case respectively. The inset of Fig. 3(b) indicates that $\theta$ increases as a stepwise exponential function, leading to a preferred alignment of the two squares. Before $t^{\prime}=0.9$, the orientation increases slowly and after that orientation angle change is noticeably accelerated. The insets of Fig. 3(c) indicate that the increase in the alignment ratio, $\lambda$, is also a stepwise exponential function. Before $t^{\prime}=2, \lambda$ increases quickly and then becomes noticeably damped. Theoretically it is not possible for two squares to reach an equilibrated configuration within a reasonable time. Due to the interaction between these two parameters, there is no possibility that $\lambda$ reaches equilibrium prior to orientation. Instead its slope changes over time. Thus in the region right of the dividing line, $\lambda$ can be described as a power law function with a changing exponent. The thermodynamically stable configuration for two squares is therefore $\theta=0^{\circ}$ and $\lambda=1$, which can be further confirmed by the net torque landscape on a square (Fig. 4). The net torque is always positive and it gradually reaches zero when the configurations of the two squares reaches $\theta=0^{\circ}$ and $\lambda=1$.

\subsection{Two rectangles}

The most stable configuration of the two squares is when they are aligned along their edges. Due to the four-fold symmetry of squares, there is no energetic preference for any of the edges. When the four-fold symmetry is broken, preference of certain edges predictably emerges. Here we demonstrate such preference by using rectangles, where $\varepsilon \neq 1$. Figures $5(a)$ and (b) shows how two rectangles with $\varepsilon=2$ gradually approach their most stable configuration. Under uniform field of any direction, both rectangles will align with their long axis in the direction of the external magnetic field. Similar to the squares shown in Fig. 1, the two rectangles will align on either their long edges or short edges. The two rectangles are initially orientated with two long 
edges fully aligned and both rectangles have $\theta=90^{\circ}$. This configuration is metastable, as a slight agitation will perturb the configuration and cause both rectangles to gradually rotate with both decreased $\theta$ and $\lambda$, which initiates the first stage motion. Unlike the dynamics observed using the squares, the decrease of $\theta$ and $\lambda$ will be accelerated as the rectangles continue to deviate further away from their initial state, and finally plateau as $\theta$ asymptotes to $13^{\circ}$ and $\lambda$ asymptotes 0 (Figs. 5(c) and (d)). Theoretically it will take infinite time for $\lambda$ to reach 0 . However, under any agitation, $\lambda$ will reach zero. The clockwise torques on both rectangles further rotate them to have a slight align on short edges and trigger the second stage motion. After which, the dynamics of the two rectangles are similar to those of two squares mentioned in Fig. 3. The first stage does not necessarily occur if the two rectangles initially align on the short edges. Notice that the orientation and alignment ratio changes shown in Figs. 5(e) and (f) are similar to those shown Figs. 3(b) and 3(c) except that for two rectangles (1) the orientation dynamics are damped instead of accelerated; (2) this occurs earlier; (3) the alignment ratio change gets damped later.

\subsection{A chain of squares}

The behavior of the two squares further indicates that a system consisting of more than two squares will stack on each other in the direction of the external field with fully aligned edges and form a chain-like structure under uniform magnetic field, similar to disks[5]. Figures 6(a) and (b) show the magnetic field distribution of the two chains consisting of disks and squares respectively, which are out of registry by half of center-to-center distance between neighboring particles and have interchain distance of $0.2 D$. The magnetic field distributions outside the 
particle arrays are similar for both cases, while those inside the particle arrays are different. The disks have higher magnetic fields along the direction of external field, and the squares have higher magnetic fields perpendicular to the direction of the external field. It is known that, chains of disk-like particles will form clusters of multiple columns due to the short-range attraction between chains that are out of registry from one another [16]. Figure 6(c) shows how the interchain distance $\sigma$ change varies the interaction between two chains. For disks, when $\sigma / \mathrm{D}$ is larger than 0.28 , the interaction between two chains is repulsive, which makes it possibile to form a single chain for dilute paramagnetic sphere system under uniform magnetic field[17]. When $\sigma / \mathrm{D}$ is smaller than 0.28 , the interaction between the chains turn to attraction and chains will form aggregate in two dimensions. However, chains consisting of squares have purely repulsion between each other regardless of interchain distance even when they are out of registry by half of interparticle distance. This predicts that there should be no two dimensional aggregate formed from squares under uniform magnetic field no matter how dense the squares are distributed. However, multiple chains segregated from each other by this repulsion force are not thermodynamically stable. Disks forming two dimensional aggregate will fall right into the groove formed by other two disks and establish a very stable triangular lattice. Due to steric hinderance, it is very energetically expensive for one disk to slide over another and create dislocation. However, chains of squares have much smoother surface which allows interchain sliding. Therefore, at short time scales, there is a possibility that chains of square particles stay segregated and form stripes. For long times, the chains will gradually slide over each other and eventually stack over to form a longer chain. 


\section{Conclusion}

We have used the LES method to calculate magnetic forces and torques on paramagnetic squares in uniform magnetic fields. From the calculated forces and torques, we have also shown the most stable configuration for systems that consist of a single square, two squares, two rectangles and chains of squares. In a uniform magnetic field, a square will orient its diagonal in the direction of the external field; two squares will fully align their edges and orient their connector vector to the direction of the external field; two rectangles will fully align their short edges and orient their connector line to the direction of the external field; and two chains of squares particles will repel each other laterally and stay segregated. The two segregated chains will eventually slide past each other and form a longer chain. These calculations reveal the thermodynamic behavior of paramagnetic squares under a uniform magnetic field. These 2-D paramagnetic squares can be extended to reveal the dynamics of 3-D paramagnetic cuboids. Paramagnetic cuboids typically exhibit four-fold symmetry[12]., with a square undersurface and a height that is smaller than the

edge of the square. The dynamics described above for squares also applies to the projection of these confined cuboids on a horizontal plane. We expect that cuboids will also form chains with their lateral surfaces fully aligned. Furthermore, interesting assemblies of magnetic particles with anisotropic magnetic field intensities can be developed. 


\section{Acknowledgements}

We gratefully thank Valter A. Ignatchenko for insightful discussions. Funding for this research was provided by the National Science Foundation under award number CBET-0955003.

\section{References}

[1] S. Melle, J.E. Martin, J. Chem. Phys., 118 (2003) 9875-9881.

[2] S.L. Biswal, A.P. Gast, Phys. Rev. E, 69 (2004) 9.

[3] P. Tierno, R. Golestanian, I. Pagonabarraga, F. Sagues, Phys. Rev. Lett., 101 (2008) 218304.

[4] R. Dreyfus, J. Baudry, M.L. Roper, M. Fermigier, H.A. Stone, J. Bibette, Nature, 437 (2005) 862-865.

[5] D.C. Li, J. Rogers, S.L. Biswal, Langmuir, 25 (2009) 8944-8950.

[6] R. Dreyfus, D. Lacoste, J. Bibette, J. Baudry, Eur. Phys. J. E, 28 (2009) 113-123.

[7] K. Zahn, R. Lenke, G. Maret, Phys. Rev. Lett., 82 (1999) 2721-2724.

[8] D. Du, D. Li, M. Thakur, S. Biswal, Soft Matter, 9 (2013) 6867-6875.

[9] D. Du, S.L. Biswal, Phys. Rev. E, 90 (2014) 033310.

[10] D. Griffiths, Introduction to Eletrodynamics, 3rd ed., Prentice Hall, 1999.

[11] D. Du, F. Toffoletto, S.L. Biswal, Phys. Rev. E, 89 (2014) 043306.

[12] K. Zhao, R. Bruinsma, T.G. Mason, Proc. Nat. Acad. Sci., 108 (2011) 2684-2687.

[13] K. Zhao, C. Harrison, D. Huse, W.B. Russel, P.M. Chaikin, Phys. Rev. E, 76 (2007) 040401.

[14] Y. Li, H. Miao, H. Ma, J.Z.Y. Chen, Soft Matter, 9 (2013) 11461-11466.

[15] V.A. Ignatchenko, I.S. Edelman, D.A. Petrov, Phys. Rev. B, 81 (2010) 054419.

[16] E.M. Furst, A.P. Gast, Physical Review E, 62 (2000) 6916.

[17] J. Byrom, P. Han, M. Savory, S.L. Biswal, Langmuir, 30 (2014) 9045-9052. 

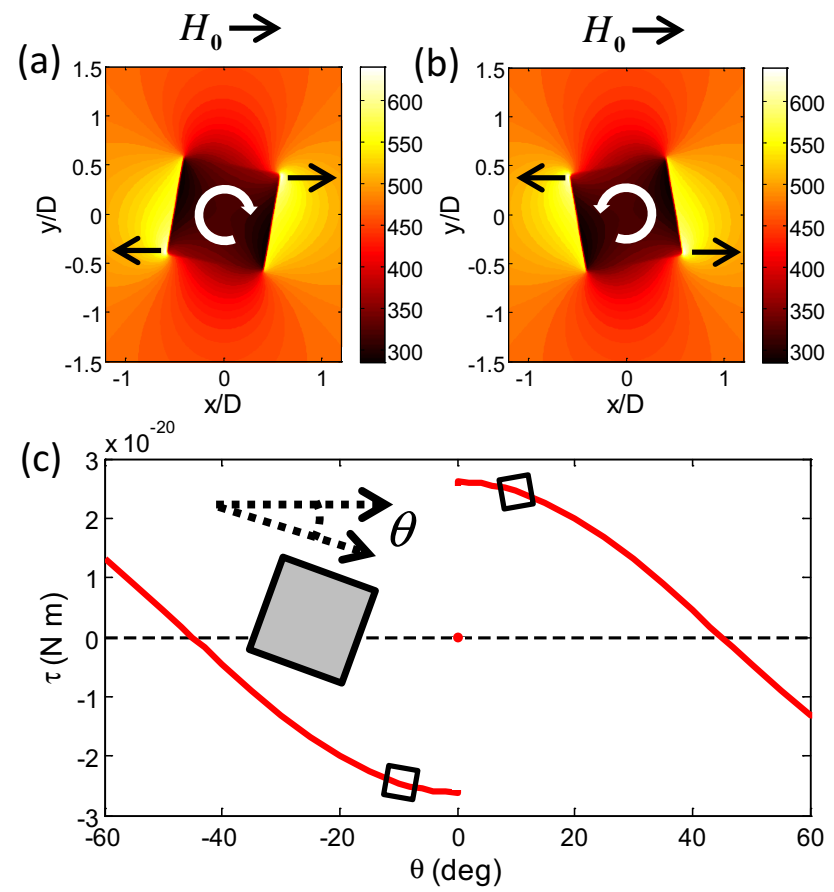

Fig. 1 A single square under a uniform magnetic field. (a) The magnetic field distribution of a square with $-10^{\circ}$ orientation. Colormaps indicate the magnetic field intensity in units of $\mathrm{A} / \mathrm{m}$. (b) The magnetic field distribution of a square with $10^{\circ}$ orientation; (c) The torque on a square with different orientations. 


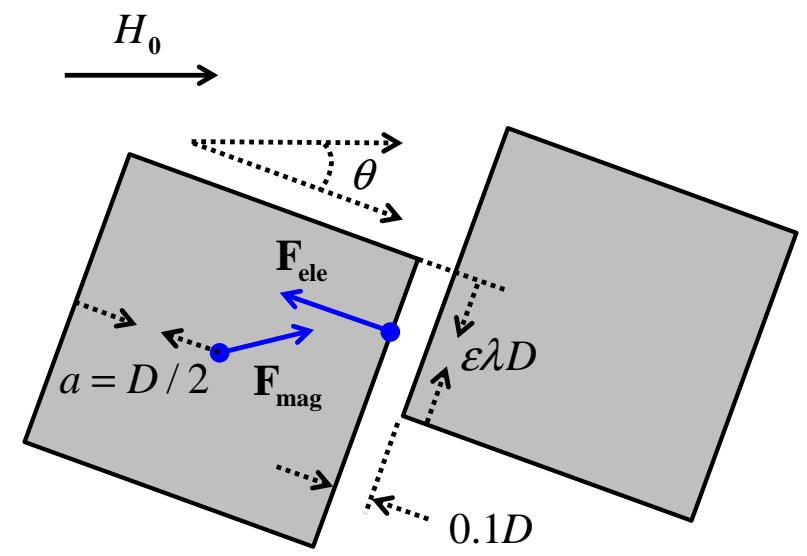

Fig. 2 Force balance and parameter illustration. 
(a)
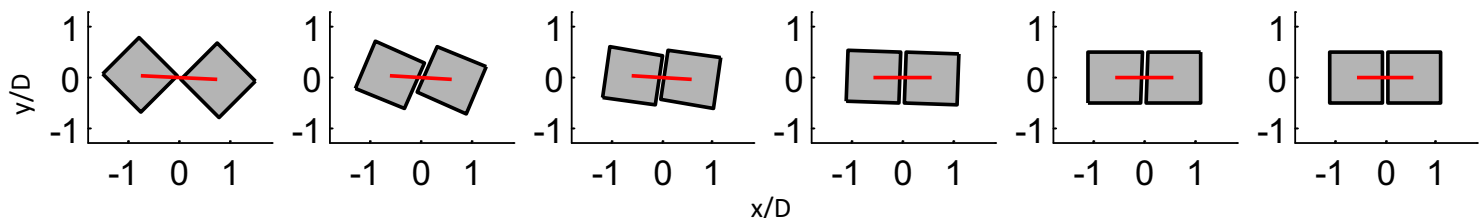

$\mathrm{O}$ Double $\mathrm{D} \times$ Double $\mathrm{B}_{0} \quad \square$ Double $\chi-$ Regular

(b)

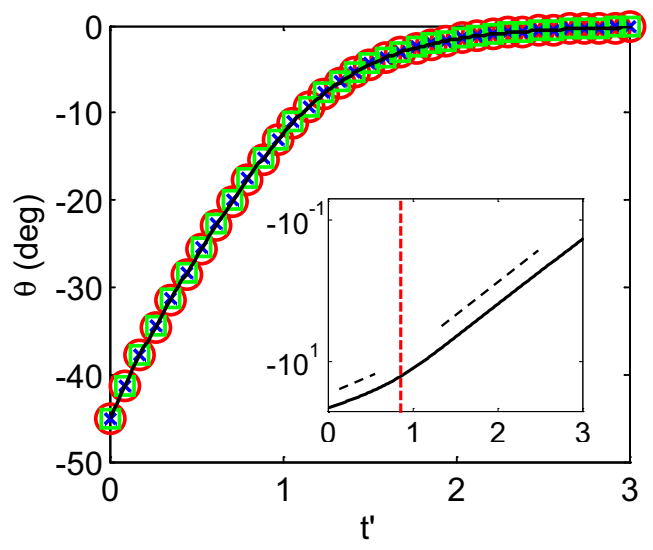

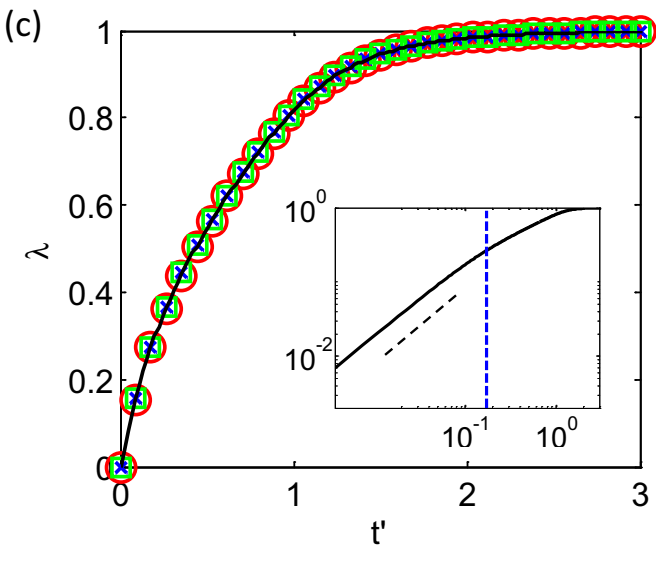

Fig. 3 Two squares under a uniform magnetic field. (a) The configuration sequence shows how the two squares move in $t^{\prime}=3$. The red lines are the connector line between the centers of two squares. (b) The orientation change over time. The inset shows the same plot with y-axis in logarithm scale. The vertical dashed line (red) divides the curve into two parts with different slopes indicated by the dashed line. (c) The alignment ratio change over time. The inset shows the same plot with both axes in logarithm scale. The vertical dashed line (blue) divides the curve into two parts with different slopes. 


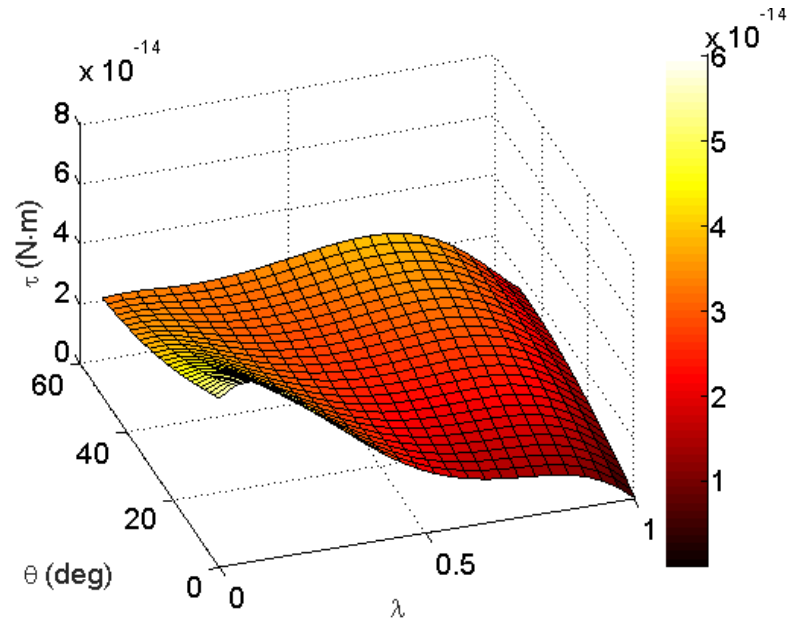

Fig. 4 The net torque on a square. 
(a)
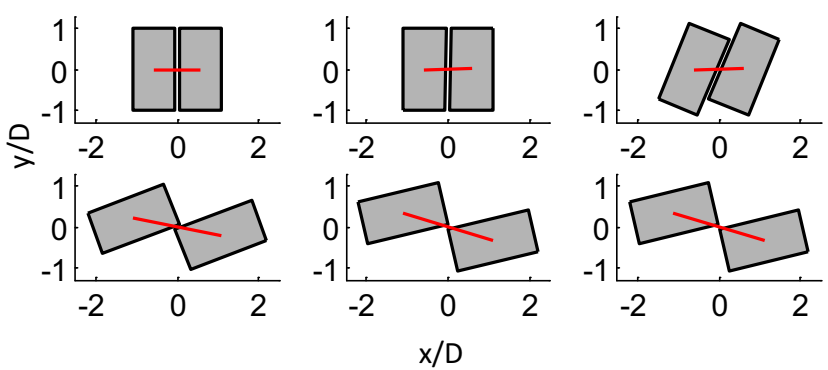

(b)
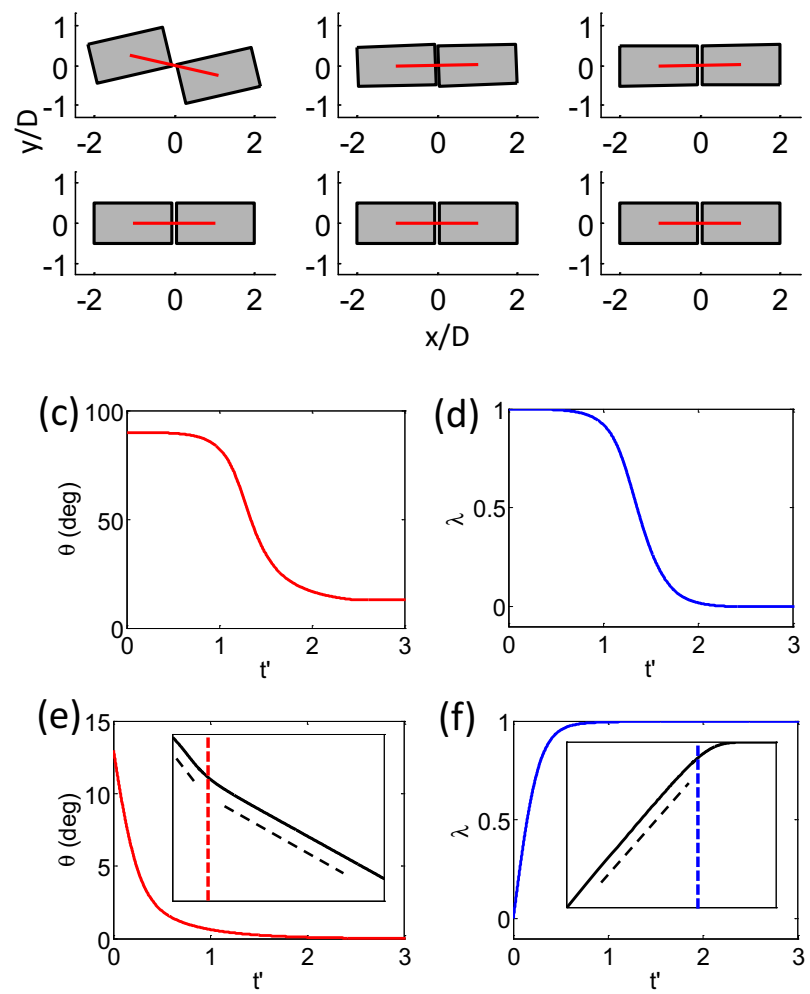

Fig. 5 Two rectangles under a uniform magnetic field. (a) The configuration sequence shows how the two rectangles move in $t^{\prime}=3$ which are initiated with fully aligned long edges (first stage). (b) The configuration sequence shows how the two rectangles move after agitation (second stage). (c) The orientation change along time for (a). (d) The alignment ratio change along time for (a). (e) The orientation change along time for (b). The inset shows the same plot with y-axis in logarithm scale. The vertical dashed line (red) divides the curve into two parts with different slopes indicated by the dashed line. The scale on y-axis is from $5 \times 10^{-3}$ to 15 . (f) The alignment ratio change along time for (b). The inset shows the same plot with both axes in logarithm scale. The scale on $y$-axis is from $5 \times 10^{-3}$ to 1 . 

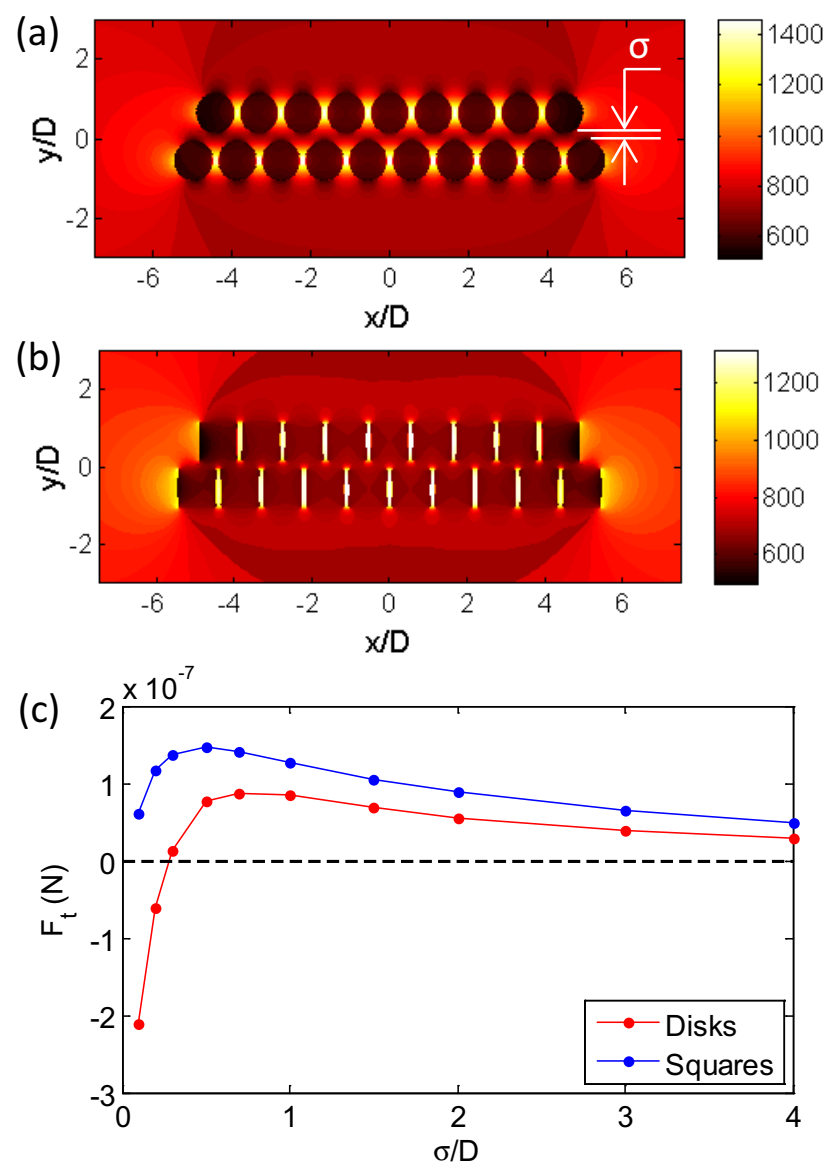

Fig. 6 Interaction between chains. (a) Magnetic field distribution of two chains of squares with $\sigma / \mathrm{D}=0.2$; (b) Magnetic field distribution of two chains of disks with $\sigma / \mathrm{D}=0.2$. Color bars in (a) and (b) indicate the strength of local magnetic field in unit of $\mathrm{A} / \mathrm{m}$. (c) The force experienced by the chain at the top. 